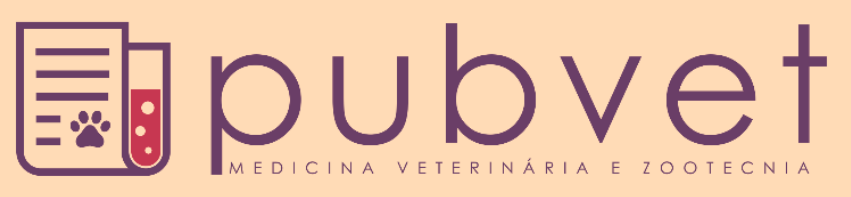

ISSN $1982-1263$

https://doi.org/10.31533/pubvet.v15n09a905.1-8

\title{
Fasceíte necrotizante: Revisão e Relato de caso em um felino
}

Samuel Otemi de Sousa Silva ${ }^{*} \bullet$, Marcelo Campos Rodrigues ${ }^{2} \bullet \bullet$, Bruno Martins Araújo $^{3} \bullet$, Breno Curty Barbosa $^{3}{ }^{\circ}$, Daniel Serafim de Andrade Rodrigues ${ }^{1} \theta$, Joana Jéssica Sousa Albuquerque $^{4}$, Thiago Martins Sousa ${ }^{4}$, Celiz de Sousa Pedrosa ${ }^{4}$

\footnotetext{
${ }^{1}$ Residente de clínica médica e cirúrgica do Hospital Veterinário Universitário, Universidade Federal do Piauí, Brasil

${ }^{2}$ Professor do departamento de clínica cirúrgica da Universidade Federal do Piauí, Brasil

${ }^{3}$ Preceptor do Hospital Veterinário Universitário - Universidade Federal do Piauí, Brasil

${ }^{4}$ Residentes em Anestesiologia do Hospital Veterinário Universitário - Universidade Federal do Piauí, Brasil

*Autor para correspondência,E-mail: samuelotemi@gmail.com
}

Resumo. Fasceíte necrosante é uma infecção súbita dos tecidos moles que progride rapidamente para a síndrome da resposta inflamatória sistêmica (SIRS), sepse e morte. O tratamento é cirúrgico. A presente revisão de literatura tem como objetivo relatar um caso de Fasceíte necrosante tipo I em um felino que sofreu trauma extenso por mordedura e expor os principais conceitos sobre Fasceíte necrosante raramente abordados em Medicina Veterinária. O seguinte caso evoluiu para anemia inflamatória grave, comprometimento dos músculos e do rim direito. Os fatores predisponentes em felinos incluem imunossupressão, gatos positivos para vírus da imunodeficiência felina (FIV), Vírus da Leucemia viral felina (FELV) e uso de corticosteroides por longos períodos. A terapia cirúrgica imediata é o tratamento recomendado na Fasceíte necrosante. Os principais agentes patológicos em gatos são Streptococcus canis e Estreptococcus sp do grupo G, Enterococcus e Escherichia sp e Pseudomonas sp, confirmados na cultura. $\mathrm{O}$ tratamento cirúrgico rápido e a antibioticoterapia adequada são essenciais para o sucesso do tratamento.

Palavras-chave: Infecção, tecidos moles, anemia inflamatória, cirúrgico

\section{Necrotizing fasciitis: Review and Caso report in feline}

Abstract. Necrotizing fasciitis is a sudden-onset soft tissue infection that progresses rapidly to systemic inflammatory response syndrome (SIRS), sepsis and death. Treatment is surgical. The present literature review aims to report a case of type I necrotizing fasciitis in a feline who suffered extensive trauma from biting, and to expose the main concepts about Necrotizing fasciitis rarely addressed in Veterinary Medicine. The following case progressed to severe inflammatory anemia, impairment of the muscles and the right kidney. Predisposing factors in felines include, immunosuppression, cats that are positive for FIV, FELV and the use of corticosteroids for long periods. Immediate surgical therapy is the treatment recommended in necrotizing fasciitis. The main pathological agents in cats is Streptococcus canis and Estreptococcus sp from group G, Enterococcus and Escherichia sp and Pseudomonas sp, confirmed in the culture. Rapid surgical treatment and adequate antibiotic therapy are essential for successful treatment.

Keywords: Infection, soft tissue, inflammatory anemia, surgical

\section{Fascitis necrotizante: Revisión y Reporte de un caso en felino}

Resumen: La fascitis necrotizante es una infección de tejidos blandos que aparece repentinamente y progresa rápidamente a síndrome de respuesta inflamatoria sistémica 
(SIRS), sepsis y muerte. El tratamiento es quirúrgico. La presente revisión de literatura tiene como objetivo reportar un caso de fascitis necrotizante tipo I en un felino que sufrió un extenso trauma por mordedura, y exponer los principales conceptos sobre la fascitis necrotizante raramente abordados en Medicina Veterinaria. El siguiente caso evolucionó a anemia inflamatoria grave, deterioro de los músculos y del riñón derecho. Los factores predisponentes en felinos incluyen, inmunosupresión, gatos que son positivos para FIV, el uso de corticosteroides por períodos prolongados. La terapia quirúrgica inmediata es el tratamiento recomendado en FN. Los principales agentes patológicos en gatos son Streptococcus canis y Estreptococcus sp del grupo G, Enterococcus y Escherichia sp y Pseudomonas sp, confirmados en el cultivo. El tratamiento quirúrgico rápido y la terapia antibiótica adecuada son esenciales para el éxito del tratamiento.

Palabras clave: Infección, tejidos blandos, anemia inflamatoria, quirúrgica

\section{Introdução}

A Fasceíte Necrotizante (FN) é uma doença de progressão rápida que se caracteriza pela necrose superficial da pele que se infiltra nos tecidos moles, tecido subcutâneo, nas fáscias e musculatura, podendo levar a morte do paciente (Sartelli et al., 2018). A FN tem origem traumática ou no póscirúrgico. Em seres humanos esta doença acomete principalmente extremidades (Fernandes et al., 2011). A FN inicia-se com necrose local que evolui para síndrome da resposta inflamatória sistêmica (SIRS), o sítio de origem passa a produzir bolhas dentro do tecido e exsudação de material purulento. Ao desenvolver SIRS e SEPSE, o paciente pode ir a óbito rapidamente por choque distributivo (Sartelli et al., 2018). O tecido necrosado e inflamado, com exsudato, promove hipoperfusão tecidual, impedindo a distribuição intralesional do antibiótico (Brady \& Otto, 2001).

Em humanos os fatores predisponentes incluem obesidade, diabetes, pessoas imunossuprimidas (Chen et al., 2020). Em animais, embora estes fatores não estejam completamente elucidados, podemos incluir o uso contínuo de corticoides, animais imunossuprimidos, presença dos vírus da imunodeficiência felina (FIV) e da leucemia felina (FELV) e outras causas de imunossupressão (Banovic et al., 2013). O vírus da FIV foi relatado em um gato com Gangrena de Founier, esta é um tipo de FN do tipo I, que consiste em tipo de FN em região genital e anorretal, desenvolveu uma rápida progressão até evoluir para choque tóxico Estreptocócico (Berube et al., 2010).

O choque tóxico é mediado por toxinas que atuam com superantígenos ativando o sistema imune, liberando de forma maciça citocinas inflamatórias, provocando o aumento permeabilidade capilar e lesão tecidual sistêmica, culminando em falência múltipla dos órgãos, tem como principais agentes o Estreptococcus pyogenes e o Estreptococcus $\beta$ hemolítico do grupo A (Antunes et al., 2011; Hoge et al., 1993). Embora esteja mais documentada em humanos esta síndrome foi descrita em um gato com Gangrena de Fournier (Berube et al., 2010).

O Estreptococcus canis do grupo G, este está presente naturalmente na cavidade nasal e sistema respiratório superior de gatos. Pacientes com presença de úlcera por herpesvírus, sinusite respiratória, imunossuprimidos, desenvolve a FN no sistema respiratório superior, causando necrose, osteolise e perfuração do seio nasal, onde, os organismos associados incluem a Clamídia sp e infecções virais como o calicívirus e herpesvírus, FIV e FELV (Pesavento et al., 2007).

Sendo pouco frequente na clínica de pequenos animais, o objetivo deste trabalho é descrever um caso de FN em um felino, abordado os aspectos clínicos, diagnósticos e terapêuticos da afecção (Greene et al., 1993; Naidoo et al., 2005).

\section{Fasceíte necrotizante}

A FN é uma doença que foi primeiramente descrita por Hipócrates em 500 a.C., em humanos, afetando as extremidades e a região ânus-genital. O termo fasciiti foi empregado no século XIX, em 1871, durante a guerra civil americana, onde, foi descrito casos de gangrena hospitalar e, em 1883, Alfred Fournier relatou fasciiti na região perineal e escroto. Em 1918, W. Pfanner foi o primeiro a sugerir o Streptococcus como causa de fasciiti durante a I guerra mundial. Em 1952, B. Wilson foi o primeiro a usar o termo fasciitis necrotizantes (FN) que ainda é utilizada até os dias de hoje ( 


\section{Fisiopatologia}

A FN inicia-se com uma celulite profunda que progride para um necrose extensa esta pode ser idiopática ou secundário ao trauma, tem como fisiopatologia a liberação massiva de citocinas pró inflamatórias após o trauma local, endarterite, formação de trombos nos vasos menores que impede o fluxo sanguíneo no tecido, presença de hemorragia multifocal, o que resulta em necrose coagulativa (Bennett et al., 2020).

A proliferação de bactérias que progridem da derme, tecido subcutâneo, fáscias e musculatura, liberando toxinas e culmina no desprendimento do tecido supra adjacente expondo a musculatura, a infecção pode evoluir para musculatura e órgãos (Nájera et al., 2019; Salcido \& Ahn, 2007). A progressão da destruição tissular tem velocidade de 2 a $3 \mathrm{~cm}$ por hora (Cunningham, 2011; Naidoo et al., 2005).

\section{Classificação da Fasceite Necrotizante}

Em humanos a FN é classificada em três grupos: O grupo I é polimicrobiana usualmente acontece após trauma, pode ser cirúrgico e geralmente o prognostico é bom. O grupo II é monomicrobiana e é causada por uma variante de Streptococcus sp do grupo A de Lancefield, conhecida como "bactéria comedoura de carne". A do tipo III é uma FN causada por Clostridium sp. causa necrose tecidual e gangrena com produção de gás normalmente causada pela bactéria Clostridium perfrigens ( $\underline{\text { Chen et al. }}$ $\underline{2020)}$.

\section{Diagnóstico}

Inicialmente os sinais clínicos pode mimetizar os sinais de outras doenças, impedindo o rápido diagnóstico, este se dá pela presença de necrose e acúmulo de liquido perifascial (Angoules et al., 2007). Aumenta-se a suspeita na presença de infecção bacteriana na cultura das amostras coletadas no trans cirúrgico e na cultura do sangue para verificar bacteremia (Cunningham, 2011; Naidoo et al., 2005). Embora, em 50\% dos pacientes humanos com FN o resultado da cultura pode dar negativo, tem-se a necessidade de começar a antibioticoterapia imediatamente (Kaul et al., 1997).

Normalmente, a radiografia é a primeiro exame a ser solicitado, onde é possível perceber edema, descontinuidade de tecidos, úlceras profundas e identificar fraturas subjacentes. No entanto, estes achados são inespecíficos. A ultrassonografia pode ajudar na identificação de tromboses, edema tecidual, presença de liquido perifascial e a tomografia computadorizada tem uma maior sensibilidade em detectar gás no tecido e corpos estranhos (Gök et al., 2017).

Nos exames laboratoriais podem estar presentes leucocitose por neutrofilia, presença de neutrófilos tóxicos, hipoalbuminemia, hipoprotenemia ou hiperproteinemia, hipercalcemia associado à hiperglicemia, aumento da fosfatase alcalina (FA), aspartato aminotransferase (AST) e creatina fosfoquinase (CK) (Naidoo et al., 2005).

Segundo Pesavento et al. (2007) e Brown \& Rogers (2001), casos de FN com neutropenia e leucopenia são frequentemente encontrados em gatos imunossuprimidos com doença viral e fúngica, a maioria dos felinos imunossuprimidos tem infecção por parvovirus felino, vírus da leucemia felina ou vírus da imunodeficiência felina (FIV).

A síndrome do choque tóxico estreptocócico pode ser diagnosticado por PCR, a presença do gene estreptocócico pirogênico produz exotoxina B é codificada no gene spedB, este está associado a mitose nos linfócitos $\mathrm{T}$ e a habilidade de aumentar a susceptibilidade para o choque endotóxico, por sua presença estável em todo grupo A de Streptococcos sp. é possível diagnosticar a presença de choque tóxico estreptocócico através de PCR, em tecidos de amostras diretas, o teste de PCR tem especificidade e sensibilidade comparado aos outros métodos de identificação do Estreptococcus sp. do grupo A produtores de toxinas (Carapetis et al., 1995; Davies et al., 1996; Louie et al., 1998).

O diagnóstico definitivo se dá por meio da histopatologia, cujos achados de necrose coagulativa, caracterizada pelo infiltrados neutrofílicos na pele, subcutâneo e fáscias, além de trombose nos vasos adjacentes confirmam o diagnóstico (Fernandes et al., 2011). 


\section{Tratamento}

O tratamento é cirúrgico, consiste no desbridamento imediato e radical a fim de retirar às fontes de infecção e toxinas bacterianas presentes (Edlich et al., 2010; Sarani et al., 2009). Casos em que há grande perda da musculatura é possível utilizar técnicas reconstrutivas com flaps e enxertos de padrão subdérmico (Wong \& Wang, 2005). No primeiro momento deve-se utilizar antibióticos de amplo espectro na associação de cefalosporinas associado à clindamicina ou metronidazol (Childers et al., 2002; Greene et al., 1993).

O diagnóstico rápido com um desbridamento cirúrgico agressivo imediato melhora o prognóstico (Nolff \& Meyer-Lindenberg, 2015; Sartelli et al., 2018). O desbridamento imediato permite a melhor perfusão do sitio da ferida, permitindo maior quantidade de antibiótico intralesional (Hussein \& Anaya, 2013). Atraso no desbridamento cirúrgico aumenta a morbidade e a mortalidade (Burch et al., 2007).

A presença de Streptococos do grupo A é um risco maior para contactantes do paciente, consequentemente a terapia preventiva deve ser recomendada para evitar surtos na saúde pública especialmente em hospitais humanos e também nos veterinários (Kaul et al., 1997). O uso de fluorquinolonas é contraindicado, e anti-inflamatórios não esterioidais é contraindicado, vários relatos indicam eficácia limitada destas drogas para prevenir síndrome do choque tóxico estreptocócico em pacientes com presença de infecções por Estreptococcus (Naidoo et al., 2005).

\section{Relato de caso}

Foi atendido no setor de emergência e cuidados intensivos do Hospital Veterinário da Universidade Federal do Piauí, (HVU), um felino, macho com idade aproximada de três anos, com massa corporal de $3 \mathrm{~kg}$. A tutora relatou que ele havia sido mordido por um cão em região abdominal há 4 horas. Ao exame clínico pôde-se observar desidratação $( \pm 8 \%)$, temperatura retal $39,4^{\circ} \mathrm{C}$, dispneia e dor em região de coluna toraco lombar. Não foi observado ferimentos na pele. No entanto, no dia seguinte, evoluiu uma necrose da pele que se estendia na região da última costela do lado direito até metade do abdômen.

Foram solicitados hemograma, bioquímico e ultrassonografia. O hemograma acusou leucocitose neutrofilica, anemia normocrômica, normocítica, não regenerativa. O bioquímico acusou azotemia. $\mathrm{Na}$ ultrassonografia foi possível observar edema e coleção de liquido perifascial e não foi observado o rim direito. Optou-se pelo tratamento clínico com associação de cefalotina sódica e metronidazol $(30 \mathrm{mg} / \mathrm{kg}$ e $15 \mathrm{mg} / \mathrm{kg}$, respectivamente, BID e IV) e cloridrato de tramadol (2,5 mg/kg/ TID/ SC).

No sétimo dia de internação, por não haver resposta clínica à medicação adotada e pelo aumento progressivo da área necrosada com exsudação purulenta, formação de solução de continuidade com a cavidade abdominal, onde, podia-se visualizar uma estrutura em região topográfica de rim, que não pôde ser identificada (Figura 1).
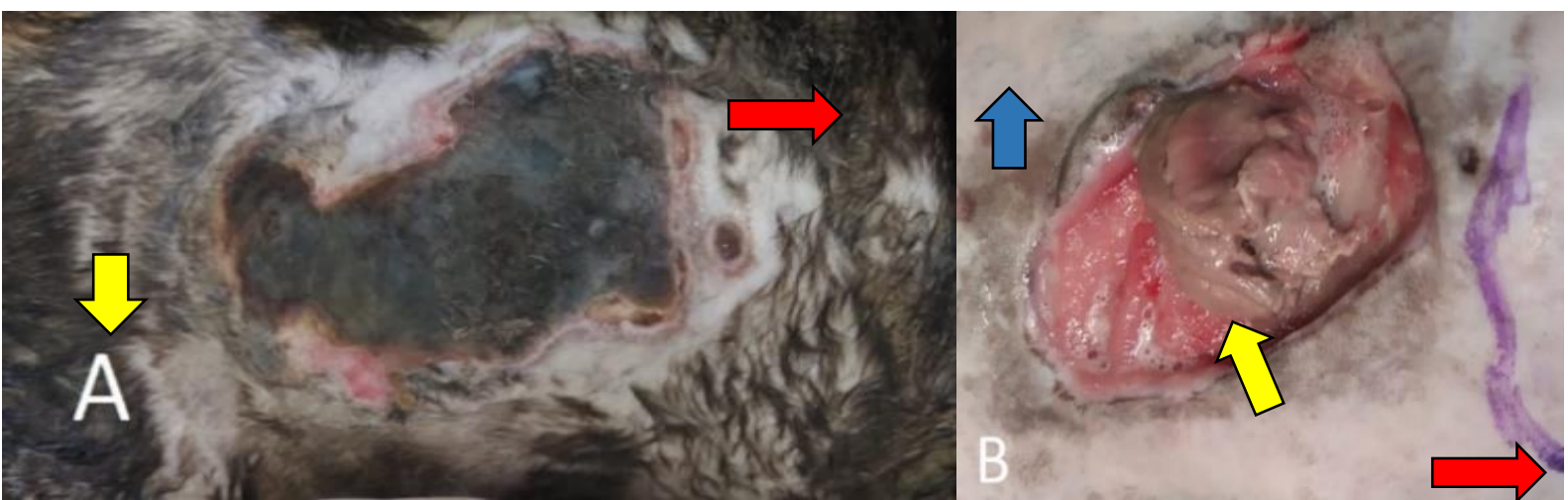

Figura 1. Faciite necrotizante em felino, atendido no HVU/UFPI, em Teresina-PI, 2020. A - Edema necrose extensa na região abdominal direita, setas indicando a posição cranial (Vermelha) e ventral (Amarela). B - Estrutura identificada, a posteriore, como sendo o rim direito saindo através da comunicação formada com o abdômen (seta amarela), seta indicando a posição cranial (Seta vermelha) e ventral do paciente (Seta Azul).

Diante da evolução do quadro, resolveu-se encaminhar o paciente para cirurgia. Dentre os exames pré-operatórios pôde-se verificar que a azotemia persistia (creatinina $-2,2 \mathrm{mg} / \mathrm{dL}$ e ureia -130 
$\mathrm{mg} / \mathrm{dL}$ ) e o hematócrito baixo (15\%). Foi realizada transfusão sanguínea, havendo uma elevação do hematócrito para $22 \%$. O paciente foi encaminhado para o centro cirúrgico.

Como medicação pré-anestésica foi utilizado midazolan $(0,2 \mathrm{mg} / \mathrm{kg} / \mathrm{IM})$ sulfato de morfina $(0,25$ $\mathrm{mg} / \mathrm{kg} / \mathrm{IM})$ e acepromazina $(0,02 \mathrm{mg} / \mathrm{kg} / \mathrm{IM})$. A indução foi alcançada com propofol $(4 \mathrm{mg} / \mathrm{kg} / \mathrm{IV})$ e a manutenção com isoflurano em circuito semiaberto com $100 \%$ de oxigênio. Após os procedimentos précirúrgicos, o paciente foi colocado em decúbito dorsal, realizou-se antissepsia do campo cirúrgico com álcool a $70 \%$ e clorexidina $2 \%$. Os panos de campos foram colocados de forma rotineira. Procedeu-se à celiotomia pré-umbilical, iniciando-se próximo ao processo xifoide, até o abdômen médio A estrutura protraída pela comunicação abdominal foi identificada como o rim direito (Figura 2). Decidiu-se realizar ureteronefrectomia desse rim, onde, por meio de dissecção o rim foi liberado de sua capsula, a artéria e veia renais foram identificadas e ligadas separadamente com fio poliadixanona 3-0. O ureter foi dissecado e ligado próximo à vesícula urinaria. Foi coletado material para cultura bacteriana e a cavidade abdominal foi lavada com solução de cloreto de sódio a $0,9 \%$ pré-aquecida $(200 \mathrm{ml} / \mathrm{kg})$. A sutura da musculatura paracostal foi realizada com fio nylon 3-0 com pontos simples separados tipo sultan, o subcutâneo com poligecaprone 4-0 com ponto tipo cushing e a pele com nylon 3 - 0 com pontos simples separados (MacPhail, 2014).

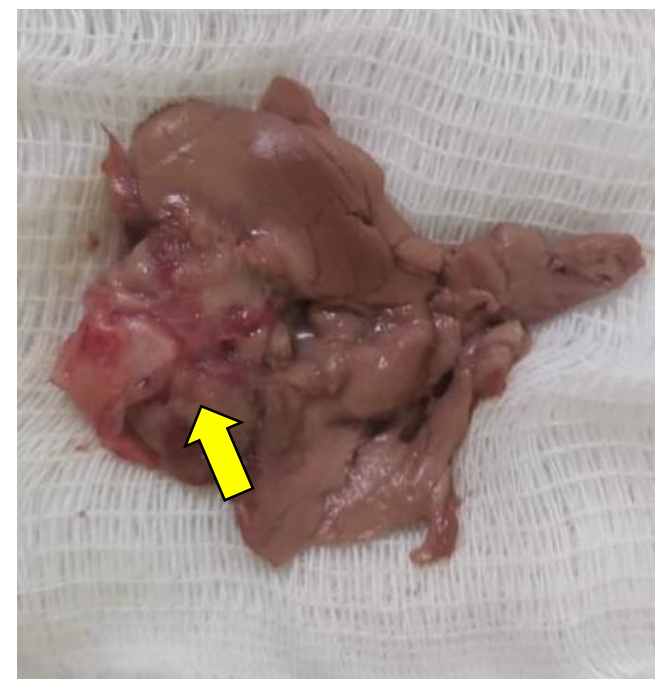

Figura 2. Aspecto macroscópico de rim direito de um felino com Faciite necrotizante, atendido no HVU/UFPI, em Teresina-PI, 2020. Seta: estrias hiperêmicas radialmente orientadas.

No pós-cirúrgico continuou-se a administração, de cefalotina sódica (30 mg/kg/BID/IV), metronidazol $(15 \mathrm{mg} / \mathrm{kg} / \mathrm{BID} / \mathrm{IV})$. Após quatro dias foi dado alta e prescrito amoxicilina com ácido clavulânico (20 mg/kg/VO, BID/ por 10 dias). Nos 15 dias após a cirurgia não houve recidiva e o sítio cirúrgico cicatrizou de forma esperada.

\section{Discussão}

Segundo Banovic et al. (2013), os sinais clínicos do paciente com FN inicia-se com hematoma extenso que evolui em um curto período para inflamação, edema, dor, calor, dependendo do tipo de FN, pode aparece bolhas (crepitação na palpação) e sinais inespecíficos como febre, sepse (taquicardia, bradicardia, hipotensão), prostração e letargia. O felino do presente relato apresentou necrose, com formação de bolhas, no entanto não evolui para sepse, apresentou mudanças de comportamento como vocalização e agitação (Banovic et al., 2013; $\underline{\text { Sartelli et al., 2018). }}$.

As bactérias Gram positivas como Estreptococcus sp. e Estaphylococcus sp. prevaleceram na cultura do material encaminhado para análise bacteriana. Segundo Mayer \& Rubin (2012), Greene et al. (1993) e Plavec et al. (2015) relataram que microrganismos como Estreptococcus do grupo G, Estreptoccocus canis, Estaphylococcus pseudointermedius são predominantes na Fasceíte necrotizante, e os microogranismos Actinobacter baumanii, Prevotella bivia, Enteroccocus faecium, Estaphylococcus 
epidermidis e Escherichia coli, Estaphylococcus hemoliticcus, Enterococcus faecalis podem desenvolver esta condição em felinos.

A FN do presente relato se enquadra na classificação presente no grupo I, pois prevaleceram na cultura Streptococcus sp e Estaphylococcus sp, sendo proveniente de trauma de mordedura e produziu gás (Chen et al., 2020). Os exames laboratoriais acusaram leucocitose neutrofílica absoluta no primeiro exame com hematócrito normal, como foi descrito por Naidoo et al. (2005). No segundo hemograma pré-cirúrgico o hematócrito já estava em $15 \%$, apresentando uma anemia normocítica, normocrômica. Segundo Schümann et al. (2005) a anemia inflamatória normalmente produz uma leucocitose, hiperproteinemia, hiperglobulinemia e anemia normocítica normocromica estas alterações do hemograma estava presente no caso relatado, a anemia pode evoluir para microcítica hipocromica pelo sequestro de ferro na corrente sanguínea.

O aumento de ecogenicidade do tecido subcutâneo e o acúmulo de líquido perifascial observados na ultrassonografia no caso relatado também foram achados citados por Gök et al. (2017). Segundo Brady \& Otto (2001), a terapia clinica tem baixa eficácia, no entanto, optou-se pelo uso da cefalotina sódica e metronidazol. De acordo com Naidoo et al. (2005) a terapia de amplo espectro deve ser preconizada associando-se cefitriaxanona com metronidazol, ou penicilina associada à clindamicina, principalmente em casos que há crepitação bolhosa. A cefalotina sódica é uma cefalosporina de primeira geração que não abrange as bactérias Gram negativas, por esta razão a terapia clinica utilizada, no presente relato não pode ser considerada antibioticoterapia de amplo espectro.

A terapia medicamentosa foi mantida por sete dias, sem sucesso e, com a evolução gradual da área de necrose optou-se pelo tratamento cirúrgico. O desbridamento cirúrgico é o meio de debelar a infecção, como preconizado por Banovic et al. (2015). Devido a comunicação com a cavidade abdominal, a celiotomia exploratória foi necessária, onde pôde-se identificar que a estrutura projetada através da área de necrose era o rim direito.

A paciente do presente relato não desenvolveu choque tóxico estreptocócico e sepse, apesar do comprometimento da musculatura de órgãos internos. A FN pode comprometer a musculatura e continuar a progredir para os órgãos internos, embora, não seja comum, o paciente não evoluir para choque tóxico estreptocócico e sepse (Bennett et al., 2020; $\underline{B r a d y ~ \& ~ O t t o, ~ 2001 ; ~ C h e n ~ e t ~ a l ., ~ 2020) . ~}$

\section{Conclusão}

A Fasceíte necrotizante é uma condição patológica que deve ser tratada de forma cirúrgica rapidamente, para que o animal não entre em sepse ou tenha seus tecidos e órgãos comprometidos. A evolução rápida torna à identificação célere e os tratamentos cirúrgicos essenciais à sobrevida do paciente.

\section{Referências bibliográficas}

Angoules, A. G., Kontakis, G., Drakoulakis, E., Vrentzos, G., Granick, M. S., \& Giannoudis, P. V. (2007). Necrotising fasciitis of upper and lower limb: a systematic review. Injury, 38(5), S18-S25.

Antunes, R., Diogo, M., Carvalho, A., Pimentel, T., \& Oliveira, J. (2011). Síndrome do choque tóxico por Streptococcus Pyogenes. Acta Medica Portuguesa, 24, 617-620.

Banovic, F., Linder, K., Boone, A., Jennings, S., \& Murphy, K. M. (2013). Cat scratch-induced P asteurella multocida necrotizing cellulitis in a dog. Veterinary Dermatology, 24(4), 463-e108.

Bennett, J. E., Dolin, R., \& Blaser, M. J. (2020). Enfermedades infecciosas. Principios y práctica. Elsevier Health Sciences.

Berube, D. E., Whelan, M. F., Tater, K. C., \& Bracker, K. E. (2010). Fournier's gangrene in a cat. Journal of Veterinary Emergency and Critical Care, 20(1), 148-154.

Brady, C. A., \& Otto, C. M. (2001). Systemic inflammatory response syndrome, sepsis, and multiple organ dysfunction. Veterinary Clinics: Small Animal Practice, 31(6), 1147-1162.

Brown, M. R., \& Rogers, K. S. (2001). Neutropenia in dogs and cats: a retrospective study of 261 cases. Journal of the American Animal Hospital Association, 37(2), 131-139. 
Burch, D. M., Barreiro, T. J., \& Vanek, V. W. (2007). Fournier's gangrene: be alert for this medical emergency. Journal of the American Academy of PAs, 20(11), 44-47.

Carapetis, J., Robins-Browne, R., Martin, D., Shelby-James, T., \& Hogg, G. (1995). Increasing severity of invasive group A streptococcal disease in Australia: clinical and molecular epidemiological features and identification of a new virulent M-nontypeable clone. Clinical Infectious Diseases, 21(5), 1220-1227.

Chen, L. L., Fasolka, B., \& Treacy, C. (2020). Necrotizing fasciitis: a comprehensive review. Wolters Kluwers Health, Nursing, 50(9), 34-40.

Childers, B. J., Potyondy, L. D., Nachreiner, R., \& Rogers, F. R. (2002). Necrotizing fasciitis: a fourteenyear retrospective study of 163 consecutive patients. The American Surgeon, 68(2), 109.

Cunningham, J. (2011). Tratado de fisiologia veterinária. Guanabara Koogan.

Davies, H. D., McGeer, A., Schwartz, B., Green, K., Cann, D., Simor, A. E., Low, D. E., \& Group, O. G. A. S. S. (1996). Invasive group A streptococcal infections in Ontario, Canada. New England Journal of Medicine, 335(8), 547-554.

Edlich, R. F., Cross, C. L., Dahlstrom, J. J., \& Long III, W. B. (2010). Modern concepts of the diagnosis and treatment of necrotizing fasciitis. The Journal of Emergency Medicine, 39(2), 261-265.

Fernandes, C., Dâmaso, C., Duarte, R., Cardoso, D. S., \& Casella, P. (2011). Necrotizing fasciitis postacute appendicitis. Acta Medica Portuguesa, 24, 621-626.

Gök, M. C., Turhan, Y., Demiroğlu, M., Kılıç, B., Akkuş, M., \& Özkan, K. (2017). Radiological assessment in necrotizing fasciitis. Medical Journal of Islamic World Academy of Sciences, 25(1), 19-21. https://doi.org/10.5505/ias.2017.59251 2017;25(1): 19-21.

Greene, C. E., Samperio, J. O., \& Gómez, J. P. (1993). Enfermedades infecciosas: Perros y gatos. Editora Interamericana.

Hakkarainen, T. W., Kopari, N. M., Pham, T. N., \& Evans, H. L. (2014). Necrotizing soft tissue infections: review and current concepts in treatment, systems of care, and outcomes. Current Problems in Surgery, 51(8), 344-362. https://doi.org/10.1067/j.cpsurg.2014.06.001.

Hoge, C. W., Schwartz, B., Talkington, D. F., Breiman, R. F., MacNeill, E. M., \& Englender, S. J. (1993). The changing epidemiology of invasive group A streptococcal infections and the emergence of streptococcal toxic shock-like syndrome: a retrospective population-based study. Jama, 269(3), 384-389.

Hussein, Q. A., \& Anaya, D. A. (2013). Necrotizing soft tissue infections. Critical Care Clinics, 29(4), 795-806.

Kaul, R., McGeer, A., Low, D. E., Green, K., Schwartz, B., \& Simor, A. E. (1997). Population-based surveillance for group A streptococcal necrotizing fasciitis: clinical features, prognostic indicators, and microbiologic analysis of seventy-seven cases. The American Journal of Medicine, 103(1), 18 24. https://doi.org/10.1016/S0002-9343(97)00160-5.

Louie, L., Simor, A. E., Louie, M., McGeer, A., \& Low, D. E. (1998). Diagnosis of group A streptococcal necrotizing fasciitis by using PCR to amplify the streptococcal pyrogenic exotoxin B gene. Journal of Clinical Microbiology, 36(6), 1769-1771.

MacPhail, C. M. (2014). Cirurgia dos sistemas reprodutivo e genital. In T. W. Fossum (Ed.), Cirurgia de pequenos animais. Elsevier.

Mayer, M. N., \& Rubin, J. E. (2012). Necrotizing fasciitis caused by methicillin-resistant Staphylococcus pseudintermedius at a previously irradiated site in a dog. The Canadian Veterinary Journal, 53(11), 1207.

Naidoo, S. L., Campbell, D. L., Miller, L. M., \& Nicastro, A. (2005). Necrotizing fasciitis: a review. Journal of the American Animal Hospital Association, 41(2), 104-109.

Nájera, F., Sánchez-Cuerda, S., Gil-Molino, M., Varela, E., Serra, R., Soler, F., Vallverdú-Coll, N., Panadero, J., Zorrilla, I., \& García, A. (2019). Fatal Streptoccocus canis Necrotizing Fasciitis and Myositis in a FreeRanging Iberian Lynx (Lynx pardinus). Journal of Wildlife Diseases, 55(3), 717-720.

Nolff, M. C., \& Meyer-Lindenberg, A. (2015). Necrotising fasciitis in a domestic shorthair cat-negative 
pressure wound therapy assisted debridement and reconstruction. Journal of Small Animal Practice, 56(4), 281-284.

Pesavento, P. A., Bannasch, M. J., Bachmann, R., Byrne, B. A., \& Hurley, K. F. (2007). Fatal Streptococcus canis infections in intensively housed shelter cats. Veterinary Pathology, 44(2), 218-221.

Plavec, T., Zdovc, I., Juntes, P., Svara, T., Ambrozic-Avgustin, I., \& Suhadolc-Scholten, S. (2015). Necrotising fasciitis, a potential threat following conservative treatment of a leucopenic cat: a case report. Veterinarni Medicina, 60(8), 460-467.

Salcido, R., \& Ahn, C. (2007). Necrotizing fasciitis: reviewing the causes and treatment strategies. Advances in Skin \& Wound Care, 20(5), 288-293. https://doi.org/10.1097/01.ASW.0000269317.76380.3b.

Sarani, B., Strong, M., Pascual, J., \& Schwab, C. W. (2009). Necrotizing fasciitis: current concepts and review of the literature. Journal of the American College of Surgeons, 208(2), 279-288.

Sartelli, M., Guirao, X., Hardcastle, T. C., Kluger, Y., Boermeester, M. A., Raşa, K., Ansaloni, L., Coccolini, F., Montravers, P., \& Abu-Zidan, F. M. (2018). 2018 WSES/SIS-E consensus conference: recommendations for the management of skin and soft-tissue infections. World Journal of Emergency Surgery, 13(1), 1-24. https://doi.org/10.1186/s13017-018-0219-9.

Schümann, K., Kroll, S., Weiss, G., Frank, J., Biesalski, H. K., Daniel, H., Friel, J., \& Solomons, N. W. (2005). Monitoring of hematological, inflammatory and oxidative reactions to acute oral iron exposure in human volunteers: Preliminary screening for selection of potentially-responsive biomarkers. Toxicology, 212(1), 10-23. https://doi.org/10.1016/j.tox.2005.03.014

Wong, C.H., \& Wang, Y.S. (2005). The diagnosis of necrotizing fasciitis. Current Opinion in Infectious Diseases, 18(2), 101-106.

Histórico do artigo:

Recebido: 9 de março de 2021

Aprovado: 7 de maio de 2021
Licenciamento: Este artigo é publicado na modalidade Acesso Aberto sob a licença Creative Commons Atribuição 4.0 (CC-BY 4.0), a qual permite uso irrestrito, distribuição, reprodução em qualquer meio, desde que o autor e a fonte sejam devidamente creditados. 